\title{
The comma-free codes with words of length two
}

\section{A.H. Ball and L.J. Cummings}

\begin{abstract}
A code not requiring a distinct symbol to separate words is called comma-free. Two codes are isomorphic if one can be obtained from the other by a permutation of the underlying alphabet. Since subcodes of comma-free codes are comma-free, we investigate only maximal comma-free codes. All isomorphism classes of maximal comma-free codes with words of length 2 are determined and a natural representative of each class is given.
\end{abstract}

\section{Introduction}

Let $\Sigma_{n}=\{0,1, \ldots, n-1\}$ be an alphabet of $n$ symbols. Let $F$ be a block code of word length $k$ over $\Sigma_{n}$. The code $F$ is said to be comma-free if whenever

$$
a_{1} \ldots a_{k} \in F \text { and } b_{1} \ldots b_{k} \in F
$$

then the words

$$
a_{2} \ldots a_{k} b_{1}, a_{3} \ldots a_{k} b_{1} b_{2}, \ldots, a_{k} b_{1} \ldots b_{k-1}
$$

are not in $F$. The words (1) are called the overlaps of $a_{1} \ldots a_{k}$ and $b_{1} \ldots b_{k}$. Alternately, a block code is comma-free if a distinct symbol is not required to separate code words in a message. Subcodes of commafree codes are comma-free.

Mathematical study of comma-free codes was initiated in 1958 by

Received 2 December 1975. 
Golomb, Gordon and Welch [2]. These authors gave an upper bound for the size $W_{k}(n)$ of a maximal comma-free code as a function of the alphabet length $n$ and the block length $k$. They conjectured that the bound was always attained when the $k$ was an odd integer. Eastman [1] found a construction resolving their conjecture 6 years later.

When the block length is even, less is known about $W_{k}(n)$. Golomb, Gordon and Welch [2] proved that for all positive integers $n$,

$$
W_{2}(n)=\left[\frac{n^{2}}{3}\right]
$$

where [•] denotes the greatest integer function. Maximal comma-free codes over a binary alphabet have been constructed for $k=2,4,6,8$, and 10 in [2], [3], and [4]. In this paper we determine all maximal comma-free codes with words of length 2 over arbitrary finite alphabets.

\section{Preliminaries}

Throughout the remainder of this paper all codes will be assumed to have words of length 2 . Every comma-free code $F$ over an alphabet $\Sigma$ induces a partition of $\Sigma$ as follows:

$$
\begin{aligned}
& A(F)=\{a \in \Sigma \mid a \text { only begins words of } F\}, \\
& B(F)=\{b \in \Sigma \mid b \text { both begins and ends words of } F\}, \\
& C(F)=\{c \in \Sigma \mid c \text { only ends words of } F\} .
\end{aligned}
$$

We say that $F$ has parometers $\left(q_{1}, q_{2}, q_{3}\right)$ if

$$
|A(F)|=q_{1},|B(F)|=q_{2},|C(F)|=q_{3},
$$

where $|X|$ always denotes the cardinality of a set $X$. Maximality of a comma-free code restricts the parameters considerably as the following lemma shows. The authors are indebted to Dr T.K. Sheng for the proof of this lemma.

LEMMA 1. Let $n$ be a positive integer. The only common positive integral solutions of the equations

$$
x y+y z+z x=\left[\frac{n^{2}}{3}\right]
$$


and

$$
x+y+z=n
$$

are

$$
\begin{aligned}
(q, q, q) & n=3 q, \\
(q+1, q, q) & n=3 q+1, \\
(q+1, q+1, q) & n=3 q+2,
\end{aligned}
$$

and their permutations.

Proof. Squaring (4) we obtain

$$
x y+y z+x z=\frac{n^{2}}{3}-\frac{1}{6}\left[(x-y)^{2}+(y-z)^{2}+(z-x)^{2}\right]
$$

We have

$$
\left[\frac{n^{2}}{3}\right]= \begin{cases}3 q^{2} & n=3 q, \\ 3 q^{2}+2 q & n=3 q+1, \\ 3 q^{2}+4 q+1 & n=3 q+2 .\end{cases}
$$

Combining (5) and (6) with (3) we obtain

$$
(x-y)^{2}+(y-z)^{2}+(z-x)^{2}= \begin{cases}0 & n=3 q, \\ 2 & \text { otherwise. }\end{cases}
$$

If $n=3 q$ then (7) implies $x=y=z=q$. Otherwise, exactly two of the differences $|x-y|,|y-z|$, and $|z-x|$ are 1 . Without loss of generality assume $x-y=1$ and further suppose $n=3 q+1$. If either $y-z=1$ or $z-y=1$ we obtain contradictions of (7) and (4) respectively. Similarly, if $z-x=1$ we contradict (7). Therefore $x-z=1$ so that (4) yields $z=q$, implying $x=q+1, y=q$, and $z=q$. The argument is similar in case $n=3 q+2$.

If $\pi$ is a permutation of the alphabet $\Sigma_{n}$ then $\pi$ induces the natural mapping $f(\pi): \Sigma_{n} \times \Sigma_{n} \rightarrow \Sigma_{n} \times \Sigma_{n}$ defined by

$$
f(\pi)(x y)=\pi(x) \pi(y),
$$

where the ordered pairs $(x, y)$ of the Cartesian product $\Sigma_{n} \times \Sigma_{n}$ are 
written as simply $x y$ for notational convenience. For any permutation $\pi$, the image of a comma-free code under $f(\pi)$ is comma-free. Two codes $F$ and $F^{\prime}$ over the same alphabet $\Sigma_{n}$ are isomorphic if there is a permutation $\pi$ of $\Sigma_{n}$ such that $f(\pi)$ is a one-to-one mapping of $F$ onto $F^{\prime}$. Isomorphic codes have the same parameters.

The mapping $x y \mapsto y x$ also preserves the comma-free property of codes with words of length 2 . The image of a code under this mapping is called its transpose. If $F$ has parameters $\left(q_{1}, q_{2}, q_{3}\right)$ then the transpose of $F$ has parameters $\left(q_{3}, q_{2}, q_{1}\right)$. An obvious necessary condition that a code with parameters $\left(q_{1}, q_{2}, q_{3}\right)$ be isomorphic to its transpose is $q_{1}=q_{3}$. For maximal comma-free codes this condition is also sufficient and in Corollary 6 we determine precisely which of these codes are isomorphic to their transposes.

LEMMA 2. Let $F$ be a comma-free code over $\Sigma_{n}$ with parameters $\left(q_{1}, q_{2}, q_{3}\right)$ where $q_{i}>1, i=1,2,3$. Choose

$$
a \in A(F), b \in B(F), \quad c \in C(F) \text {. }
$$

If all words of $F$ containing at least one of $a, b$, or $c$ are deleted then the remaining words form a comma-free code $F_{1}$ and

$$
\left|F_{1}\right| \geq|F|-2 n+3
$$

Proof. There are at most $q_{1}+q_{2}$ words of the form $x c$ in $F$ since $x$ can be chosen only from $A(F)$ or $B(F)$. There are at most $q_{2}+q_{3}$ words of the form ay in $F$ since $y$ can be chosen only from $B(F)$ or $C(F)$. There are at most $q_{3}$ words of the form by since words $b_{1} b_{2}$ with $b_{1}, b_{2} \in B(F)$ cannot appear in comma-free codes. Similarly there are at most $q_{1}$ words of the form $x b$ in $F$. Thus, at most

$$
\left(q_{1}+q_{2}\right)+\left(q_{2}+q_{3}-1\right)+\left(q_{1}-1\right)+\left(q_{3}-1\right)=2\left(q_{1}+q_{2}+q_{3}\right)-3=2 n-3
$$

words of $F$ are deleted. Therefore, $\left|F_{1}\right| \geq|F|-2 n+3$. 


\section{Results}

For notational convenience we write for integers $x, y, a, b$,

$$
x y \equiv a b(\bmod 3)
$$

if $x \equiv a(\bmod 3)$ and $y \equiv b(\bmod 3)$

THEOREM 3. If $n \equiv 0(\bmod 3)$ then every maximal comma-free code with words of length 2 over $\Sigma_{n}$ is isomorphic to

$$
c_{n}=\left\{x y \mid x y \equiv 01,02 \text {, or } 12(\bmod 3) \text { where } x, y \in \Sigma_{n}\right\}
$$

or $c_{n}$ tronsposed.

Proof. Let $F$ be a maximal comma-free code over $\Sigma_{3 q}$. The proof is by induction on $q$.

If $q=1$ then $F$ has size 3 by (2). The maximality of $F$ implies every element of $\Sigma_{3}$ appears in some word of $F$. If 0 does not begin a word of $F$ then consider the transpose of $F$ instead. We may suppose $F$ contains a word $x y$ with $x=0$. Since $F$ is comma-free, $y$ cannot be 0 ; so $y$ is either 1 or 2 . Therefore $F$ contains either 01 or 02 .

If $F$ contains both 01 and 02 then $F$ contains either 12 or 21 . This follows because then $F$ cannot contain either 10 or 20 and remain comma-free, leaving only 12 or 21 as possibilities. If $F$ contains 21 then the permutation (12) of $\Sigma_{3}$ induces an isomorphism of $F$ and $C_{3}$. Otherwise, $F$ is identically $C_{3}$.

Now suppose $F$ contains only 01 . The remaining words of $F$ are among 20, 12 , and 21 . Since the pair 12 and 21 cannot appear in a comma-free code, $F$ must contain 20 . But $\{01,20,12\}$ is not commafree because 12 is an overlap of 01 and 20 . Therefore, $F=\{01,20,21\}$. The permutation (012) of $\Sigma_{3}$ induces an isomorphism of $F$ and $C_{3}$.

The argument is similar if $F$ contains only 02 .

Now suppose $q>1$. By Lemma $I, F$ has parameters $(q, q, q)$. Choosing $a, b, c$ as in (8) and deleting words of $F$ containing them, we 
obtain a comma-free subcode $F_{1}$ of $F \cdot F_{1}$ has parameters $(q-1, q-1, q-1)$. Since $|F|=3 q^{2}$, (9) implies $\left|F_{1}\right| \geq 3(q-1)^{2}$. But the number of words in any maximal comma-free code over $\Sigma_{3 q-3}$ is $3(q-1)^{2}$ by (2). Therefore $F_{1}$ is a maximal comma-free code. By induction, there is an isomorphism $f\left(\pi_{1}\right)$ of $F_{1}$ and $C_{3 q-3}$ or $C_{3 q-3}$ transposed. The permutation $\pi_{1}$ of $\Sigma_{3 q-3}$ may be extended to a permutation $\pi$ of $\Sigma_{3 q}$ by defining

$$
\pi(a)=3 q-3, \pi(b)=3 q-2, \pi(c)=3 q-1 .
$$

We verify that $f(\pi)$ is an isomorphism of $F$ and $C_{3 q}$ as follows.

For words containing $a, b$ or $c$ we have

$$
\begin{aligned}
& f(\pi)(a y)=(3 q-3) \pi(y), f(\pi)(x b)=\pi(x)(3 q-2), \\
& f(\pi)(b y)=(3 q-2) \pi(y), f(\pi)(x c)=\pi(x)(3 q-1) .
\end{aligned}
$$

If, for example,

$$
\pi(x)(3 q-1)=\dot{\pi}\left(x^{\prime}\right)(3 q-1)
$$

obviously $x=x^{\prime}$. If, say,

$$
\pi(x)(3 q-1)=(3 q-3) \pi(y),
$$

then $x=a$ and $y=c$. The other cases are similar. Therefore $f(\pi)$ is one-to-one.

THEOREM 4. If $n \equiv I$ ( $\bmod 3$ ) then every maximal comma-free code with words of length 2 over $\Sigma_{n}$ is isomorphic to $c_{n}$,

$$
D_{n}=\left\{x y \mid x y \equiv 10,02 \text {, or } 12(\bmod 3) \text { where } x, y \in \Sigma_{n}\right\} \text {, }
$$

or one of their transposes.

Proof. Let $F$ be a maximal comma-free code over $\Sigma_{3 q+1} \cdot$ The proof is by induction on $q$.

If $q=1$ then $F$ has size 5 by (2). By Lemma $I$ we may suppose $F$ has parameters $(2,1,1),(1,2,1)$ or $(1,1,2)$. If $F$ has parameters $(1,1,2)$ then the transpose of $E$ would have parameters $(2,1,1)$. 
If $F$ has parameters $(2,1,1)$ and $a \in A(F)$ then there are at most 2 words in $F$ containing $a$. When the words containing $a$ are deleted from $F$ the resulting comma-free code $F_{1}$ has at least 3 words. Therefore (2) implies that $F_{1}$ contains exactly 3 words and so is a maximal comma-free code. By Theorem 3 there is an isomorphism $f\left(\pi_{1}\right)$ of $F_{1}$ and $C_{3}$ or $C_{3}$ transposed. The permutation $\pi_{1}$ of $\Sigma_{3}$ can be extended to a permutation $\pi$ of $\Sigma_{4}$ by setting $\pi(a)=3$. For the two words of the form $a x$ in $F$ we obtain

$$
f(\pi)(a x)=3 \pi(x)
$$

so that $f(\pi)$ is one-to-one.

If $F$ has parameters $(1,2,1)$ and $b \in B(F)$ then deletion of words containing $b$ leads again to an isomorphism of the remaining subcode with $c_{3}$ or its tranpose. The permutation (01) induces an isomorphism of $C_{3}$ and the subcode $\{10,02,12\}$ of $D_{4}$. The permutation (02) induces an isomorphism of $C_{3}$ and its transpose. Extending the appropriate permutation to a permutation $\pi$ of $\Sigma_{4}$ by assigning $\pi(b)=3$, we obtain an isomorphism of $F$ and $D_{4}$.

Now suppose $q>1$. By Lemma $1, F$ has parameters $(q+1, q, q)$, $(q, q+1, q)$, or $(q, q, q+1)$. If $F$ has parameters $(q, q, q+1)$ then $F$ transposed has parameters $(q+1, q, q)$.

Assume $F$ has parameters $(q+1, q, q)$. Choosing $a, b$, and $c$ as in (8) and deleting the words containing them, we obtain a comma-free subcode $F_{1}$ of $F$. The code $F_{1}$ has parameters $(q, q-1, q-1)$. Since $F$ has $3 q^{2}+2 q$ words by (2), the inequality (9) implies $\left|F_{1}\right| \geq 3 q^{2}-4 q+1$. But the size of a maximal comma-free code over $\Sigma_{3 q-2}$ is $3 q^{2}-4 q+1$ by (2) and (6). Therefore $F_{1}$ is a maximal comma-free code over $\Sigma_{3 q-2}$

By induction, there is a permutation $\pi_{1}$ of $\Sigma_{3 q-2}$ inducing an 
isomorphism of $F_{1}$ with $C_{3 q-2}, D_{3 q-2}$, or one of their transposes. But $D_{3 q-2}$ and its transpose have parameters $(q-1, q, q-1)$ and $F_{1}$ has parameters $(q, q-1, q-1)$. Therefore $F_{1}$ is isomorphic with $C_{3 q-2}$. We extend $\pi_{1}$ to a permutation $\pi$ of $\Sigma_{3 q+1}$ by defining

$$
\pi(a)=3 q, \pi(b)=3 q-2, \pi(c)=3 q-1 .
$$

It is readily verified that $f(\pi)$ is an isomorphism of $F$ and $C_{3 q+1}$.

Now assume $F$ has parameters $(q, q+1, q)$. As above we obtain a subcode $F_{1}$ of $F$ which is maximal and comma-free over $\Sigma_{3 q-2}$ but with parameters $(q-1, q, q-1)$ in this case. This time induction implies $F_{1}$ is isomorphic to $D_{3 q-2}$ or its transpose via a permutation $\pi_{1}$ of $\Sigma_{3 q-2} \cdot$ The assignments

$$
\pi(a)=3 q-1, \pi(b)=3 q, \pi(c)=3 q-2,
$$

extend $\pi_{1}$ to a permutation $\pi$ of $\Sigma_{3 q+1}$ which induces an isomorphism of $F$ and $D_{3 q+1}$.

THEOREM 5. If $n \equiv 2(\bmod 3)$ then every maximaz comma-free code with words of length 2 over $\Sigma_{n}$ is isomorphic to $C_{n}$,

$$
E_{n}=\left\{x y \mid x y \equiv 01,02 \text {, or } 21(\bmod 3) \text { where } x, y \in \Sigma_{n}\right\} \text {, }
$$

or one of their transposes.

Proof. Let $F$ be a maximal comma-free code over $\Sigma_{3 q+2}$. The proof is by induction on $q$.

If $q=1$ then $F$ has size 8 by (2). By Lemma 1, we may suppose $F$ has parameters $(2,2,1),(2,1,2)$, or $(1,2,2)$. If $F$ has parameters $(1,2,2)$ then the transpose of $F$ would have parameters $(2,2,1)$.

If $F$ has parameters $(2,2,1)$ and $b \in B(F)$ we delete the words of $F$ containing $b$ to obtain a subcode $F_{1}$ with parameters $(2,1,1)$. There can be at most 2 words of the form $x b$ in $F$ and only one of the form by. Therefore, $F_{1}$ contains at least 5 words. But $F_{1}$ contains 
at most 5 words by (2). By Theorem 4, $F_{1}$ is isomorphic to $C_{4}$ since $C_{4}$ transposed has parameters $(1,1,2)$ and both $D_{4}$ and its transpose have parameters $(1,2,1)$.

The permutation inducing this isomorphism may be extended to a permutation $\pi$ of $\Sigma_{5}$ by the assignment $\pi(b)=4$. This induces an isomorphism of $F$ and $C_{5}$.

On the other hand, if $F$ has parameters $(2,1,2)$ and $c \in C(F)$ then deletion of words containing $c$ leaves a subcode $F_{1}$ also having parameters $(2,1,1)$ and isomorphic to $C_{4}$. This time, nowever, $F$ cannot be isomorphic to $C_{5}$ because the assignment $\pi(c)=4$ could not induce correspondences with the words 04,42 , and 34 in $C_{5}$. Since $C_{4}$ and $E_{4}$ are isomorphic via the permutation $\pi=(12)$, defining $\pi(c)=4$ will induce an isomorphism of $E$ and $E_{5}$.

Now suppose $q>1$. By Lemma $1, F$ has parameters $(q+1, q+1, q)$, $(q+1, q, q+1)$ or $(q, q+1, q+1)$. If $F$ has parameters $(q, q+1, q+1)$ then $F$ transposed has parameters $(q+1, q+1, q)$.

Assume $F$ has parameters $(q+1, q+1, q)$. Choosing $a, b$, and $c$ as in ( 8$)$ and deleting the words containing them, we obtain a comma-free subcode $F_{1}$ of $F$. The code $F_{1}$ has parameters $(q, q, q-1)$. By (9), $\left|F_{1}\right| \geq 3 q^{2}-2 q$. But the size of a maximal comma-free code over $\Sigma_{3 q-1}$ is $3 q^{2}-2 q$ by (2). Therefore $F_{I}$ is isomorphic to $c_{3 q-1}, E_{3 q-1}$, or one of their transposes.

But $E_{3 q-1}$ and its transpose have parameters $(q, q-1, q)$ while $c_{3 q-1}$ has parameters $(q, q, q-1)$. Therefore $F_{1}$ is isomorphic to $c_{3 q-1}$, via a permutation $\pi_{1}$ of $\Sigma_{3 q-1}$. Extending $\pi_{1}$ to a permutation $\pi$ of $\Sigma_{3 q+2}$ by defining

$$
\pi(a)=3 q, \pi(b)=3 q+1, \pi(c)=3 q-1,
$$

we obtain an isomorphism of $F$ and $C_{3 q+2}$. 
On the other hand, if $F$ has parameters $(q+1, q, q+1)$ similar arguments lead to a subcode $F_{1}$ isomorphic to either $E_{3 q-1}$ or its transpose. Extending the permutation $\pi_{1}$ of $\Sigma_{3 q-1}$ involved to $\Sigma_{3 q+2}$ by defining

$$
\pi(a)=3 q, \pi(b)=3 q-1, \pi(c)=3 q+1,
$$

we obtain an isomorphism of $F$ with either $E_{3 q+2}$ or its transpose.

COROLLARY 6. The maximal comma-free codes with words of length 2 which are isomorphic to their transposes are $C_{3 q}, D_{3 q+1}$, and $E_{3 q+2}$ for each positive integer $q$.

Proof. The necessary condition that $q_{1}=q_{3}$ if the code has parameters $\left(q_{1}, q_{2}, q_{3}\right)$ is also sufficient. This follows because each of the $q_{1}$ elements of the set $A$ appear with equal frequency in the code and similarly for the set $C$.

\section{References}

[1] Willard L. Eastman, "On the construction of comma-free codes", IEEE Trans. Information Theory IT-11 (1965), 263-267.

[2] S.W. Golomb, Basil Gordon and L.R. Welch, "Comma-free codes", Conad. J. Math. 10 (1958), 202-209.

[3] B.H. Jiggs, "Recent results in comma-free codes", Canad. J. Math. 15 (1963), 178-187.

[4] Yoji Niho, "On maximal comma-free codes", IEEE Trans. Information Theory IT-19 (1973), 580-581.

Department of Mathematics, University of Newcastle, Newcastle, New South Wales;

Faculty of Mathematics, University of Waterloo, Waterloo, Ontario, Canada. 